\title{
Wound healing in older oncologic patients
}

\author{
Jakub Kenig \\ Department of General, Gastrointestinal, Oncologic Surgery and Transplantology, I Chair of General Surgery, \\ Jagiellonian University Medical College, Krakow, Poland
}

\begin{abstract}
The wound itself, along with lymphatic oedema, nausea and vomiting, fatigue and psychological stress, is listed as one of the five factors most negatively influencing cancer patients. Current oncologic treatment strategies are based on multimodal protocols including surgery, radiation and chemotherapeutic regimens. Thus, in oncology we can have a patient with a surgical wound, a wound that is a complication from radio- or chemotherapy, or a wound due to cancer progression. With increasing age, skin functions deteriorate due to the quantitative and qualitative changes of skin cells. Despite this, it seems that wound healing in healthy older patients is only delayed, but not completely defective. This effect is clinically apparent by the age of 60 and becomes significant at the age of 70. In turn, scar maturation improves in comparison with young individuals. However, the skin alone is more susceptible to injury in older patients. As older patients are qualified for complex oncologic treatment more and more often, wound healing in older cancer patients has become a matter of critical importance.
\end{abstract}

Key words: older oncologic patients, elderly, wound healing

About one-half of cancer cases and two-thirds of cancer deaths occur in patients aged 65 years or older [1]. As was mentioned in the previous papers, the incidence of cancer increases with age, so the number of older patients with cancer is expected to rise further in the coming years. The fastest growing segment of this population, those over 85 years of age, is also the cohort with the highest incidence of chronic wounds. As they are undergoing complex oncologic treatment more and more often, there is also a significant increased risk of impaired wound healing among this population [2, 3].

Annually it is estimated that worldwide prevalence of surgical wounds is 114,271 thousand (with average healing time app. 10-14 days and annual growth 3.6\%), of chronic wounds 40,400 thousand (with indefinite healing time and annual growth 7.6\%) and of complicated skin cancer 103 thousand (with average healing time 28 days and annual growth 3.1\%) [4]. In this context, wound healing in older cancer patients becomes a matter of critical importance. Moreover, the wound, along with lymphatic oedema, nausea and vomiting, fatigue and psychological stress, is listed as one of the five factors most negatively influencing cancer patients. The pain, smell, infection, exudate, bleeding, cosmetic appearance with the wound have a direct effect on feelings of fear, shame, uncertainty, inconvenience, isolation, loss of function and low self-esteem in cancer patients [5].

Current oncologic treatment strategies are based on multimodal protocols including surgery, radiation and chemotherapeutic regimens. Thus, in oncology we can have the patient with a surgically created wound, a wound that is a complication of radio- or chemotherapy, or a wound due to cancer progression. The last type, a malignant wound, affects app. $5 \%$ of oncologic patients and app. $10 \%$ of patients with metastasis [6].

Normal wound healing is an innate immune response to injury with the aim of restoring anatomic and functional tissue integrity. It is a tightly regulated series of processes involving

\section{How to cite:}

Kenig J. Wound healing in older oncologic patients. NOWOTWORY J Oncol 2021; 71: 49-51. 
numerous cell types, mediators and proteolytic enzymes. The first phase - haemostasis - starts immediately after injury, followed by distinct, but overlapping, phases of inflammation (usually lasting for 2-4 days), proliferation (begins within 72 hours of injury and lasts for about 14 days) and tissue remodelling (starting at around day 8, which can persist for 1 year or longer). During haemostasis, injured micro-vessels constrict, a coagulation cascade becomes activated, platelets aggregate, and a fibrin clot serving as a provisional matrix, is formed [7]. The inflammatory phase is characterised by the sequential infiltration of neutrophils, monocytes and lymphocytes. Within 24-48 hours, monocytes migrate into the wound and differentiate into mature macrophages - the key regulator of this phase. Next is the proliferative phase, which is characterised by the replacement of the provisional fibrin matrix with granulation tissue, angiogenesis and transformation of fibroblasts into myofibroblasts; this, in turn, will cause contraction of the wound and matrix remodeling [8].

With increasing age, skin functions deteriorate due to quantitative and qualitative changes in the skin cells. The most important changes are as follows:

- decreased proliferation of keratinocytes,

- increased keratinocyte migration time,

- decrease in the number of macrophages and in fibroblasts,

- atrophy of the epidermis,

- flattening of the dermal-epidermal junction,

- reduced, disorganised microcirculation,

- reduced vascular response,

- decrease in Langerhans cells and melanocytes.

Despite this, it seems that wound healing in healthy older patients is only delayed, but not defective. This effect is clinically apparent by the age of 60 and becomes significant at the age of 70. In turn, scar maturation improves in comparison with young individuals [9]. The lack of consensus on this matter is mainly due to the fact that most of the studies use the chronological age and not the biological. As was discussed in the previous papers, the older population is a very heterogenic cohort and its heterogeneity increases with age. However, the skin alone is more susceptible to injury in older patients. An ex-vivo model demonstrated that the application of a compressive load to aged skin resulted in sub-epidermal separation and altered orientation of the collagen fibres, similar to that seen in patients with pressure ulcers [10].

Multiple general processes have been associated with age that influence wound healing. Among others, malnutrition, decline of sex and steroid hormones, immobilisation, medication, obesity and comorbidities such as diabetes, renal failure, peripheral arterial disease and chronic venous insufficiency are the most often studied factors [11-15]. Between the ages of 68 and 78, a healthy person loses approximately 1\% of fat-free mass per year. A reduced perception of hunger, early satiety and changes in the hormonal mediators associated with energy balance may additionally influence the process. This loss translates to a 3-fold loss of strength. The combination of sarcopenia, functional decline, malnutrition and the inability of aged skin to distribute a pressure load substantially increases the risk of impaired wound healing, formation of chronic wound and pressure ulcers [16].

The timing of surgical intervention in relation to radio- or chemotherapy is fundamentally important as regards surgical wound healing. Radiotherapy has a significant role in the local control of cancer; however, because it non-specifically damages adjacent tissue, it can further complicate wound healing. This depends mainly on the total amount of radiation exposure as well as the timing and overall duration of treatment. In cases of doses larger than $50 \mathrm{~Gy}$, or treatments given less than 3 weeks before surgery, a significant increase in wound complications may be observed. In turn, chemotherapeutic agents interfere with many pathways that are essential to wound healing: they can delay cell migration, impair cell proliferation and reduce angiogenesis and matrix formation. Furthermore, they weaken the immune system and thereby increase the risk of infection [17]. As far as medication is concerned, we cannot forget systemic glucocorticoids, which may inhibit wound repair by anti-inflammatory effects suppression of fibroblast proliferation and collagen synthesis [18].

The research interest in wound healing in cancer patients will continue to grow; not only in the context of an ageing population but also because tumours appear to behave similar to wounds that fail to heal. Many cellular and molecular similarities have been studied in recent years that indicate multiple shared mechanisms between wounds and tumours; they differ only in that one is well regulated during wound healing and the other dysregulated during cancer growth/ metastasis. Normal wound repair has a resolution phase, in turn, cancer cells behave more similar to a chronic wound, which has no such phase [19].

\section{Conflict of interest: none declared}

\section{Jakub Kenig \\ Jagiellonian University Medical College \\ I Chair of General Surgery \\ Department of General, Oncologic, Gastrointestinal Surgery and Transplantology \\ ul. Jakubowskiego 2 \\ 30-688 Kraków, Poland \\ e-mail:jkenig@cm-uj.krakow.pl}

Received and accepted: 29 Dec 2020

\section{References}

1. Hurria A, Gupta S, Zauderer M, et al. Developing a cancer-specific geriatric assessment: a feasibility study. Cancer. 2005; 104(9): 1998-2005, doi: 10.1002/cncr.21422, indexed in Pubmed: 16206252.

2. Sen CK, Gordillo GM, Roy S, et al. Human skin wounds: a major and snowballing threat to public health and the economy. Wound Repair Regen. 2009; 17(6): 763-771, doi: 10.1111/j.1524-475X.2009.00543.x, indexed in Pubmed: 19903300. 
3. Margolis D. The Incidence and Prevalence of Pressure Ulcers among Elderly Patients in General Medical Practice. Ann Epidemiol. 2002; 12(5): 321-325, doi: 10.1016/s1047-2797(01)00255-1.

4. MedMarket Diligence, LLC; Report \#S190 and Report \#S249 (Worldwide Wound Management, Forecast to 2024: Established and Emerging Products, Technologies and Markets in the Americas, Europe, Asia/ Pacific and Rest of World. December 2015 Report \#S251).

5. Lindsay E, Renyi R, Wilkie P, et al. Patient-centred care: a call to action for wound management. J Wound Care. 2017; 26(11): 662-677, doi: 10.12968/jowc.2017.26.11.662, indexed in Pubmed: 29131749.

6. Hawthorn M. Caring for a patient with a fungating malignant lesion in a hospice setting: reflecting on practice. Int J Palliat Nurs. 2010; 16(2): 70-2, 74, 76, doi: 10.12968/ijpn.2010.16.2.46752, indexed in Pubmed: 20220684.

7. Shaw TJ, Martin P.Wound repair at a glance. J Cell Sci. 2009; 122(Pt 18): 3209-3213, doi: 10.1242/jcs.031187, indexed in Pubmed: 19726630.

8. Cañedo-Dorantes L, Cañedo-Ayala M. Skin Acute Wound Healing: A Comprehensive Review. Int J Inflam. 2019; 2019: 3706315, doi: 10.1155/2019/3706315, indexed in Pubmed: 31275545.

9. Wicke C, Bachinger A, Coerper S, et al. Aging influences wound healing in patients with chronic lower extremity wounds treated in a specialized Wound Care Center. Wound Repair Regen. 2009; 17(1): 25-33, doi: 10.1111/j.1524-475X.2008.00438.x, indexed in Pubmed: 19152648

10. Stojadinovic O, Minkiewicz J, Sawaya A, et al. Deep tissue injury in development of pressure ulcers: a decrease of inflammasome activation and changes in human skin morphology in response to aging and mechanical load. PLoS One. 2013; 8(8): e69223, doi: 10.1371/journal. pone.0069223, indexed in Pubmed: 23967056.
11. Hardman MJ, Ashcroft GS. Estrogen, not intrinsic aging, is the major regulator of delayed human wound healing in the elderly. Genome Biol. 2008; 9(5): R80, doi: 10.1186/gb-2008-9-5-r80, indexed in Pubmed: 18477406 .

12. Gosain A, DiPietro LA. Aging and wound healing. World J Surg. 2004 28(3): 321-326, doi: 10.1007/s00268-003-7397-6, indexed in Pubmed: 14961191.

13. Wicke $C$, Bachinger $A$, Coerper $S$, et al. Aging influences wound healing in patients with chronic lower extremity wounds treated in a specialized Wound Care Center. Wound Repair Regen. 2009; 17(1): 25-33, doi: 10.1111/j.1524-475X.2008.00438.x, indexed in Pubmed: 19152648.

14. DiPietro LA. Wound healing: the role of the macrophage and other immune cells. Shock. 1995; 4(4): 233-240, indexed in Pubmed: 8564549.

15. Ashcroft G, Mills $S$, Ashworth J. Ageing and wound healing. Biogerontology. 2002; 3(6): 337-345

16. Roberts SB, Rosenberg I. Nutrition and aging: changes in the regulation of energy metabolism with aging. Physiol Rev. 2006; 86(2): 651-667, doi: 10.1152/physrev.00019.2005, indexed in Pubmed: 16601270.

17. Sgonc R, Gruber J. Age-Related Aspects of Cutaneous Wound Healing: A Mini-Review. Regenerative and Technological Section / Mini-Review. Gerontology. 2013; 59: 159-164.

18. Guo S, Dipietro LA. Factors affecting wound healing. J Dent Res. 2010; 89(3): 219-229, doi: 10.1177/0022034509359125, indexed in Pubmed: 20139336.

19. Schäfer M, Werner S. Cancer as an overhealing wound: an old hypothesis revisited. Nat Rev Mol Cell Biol. 2008; 9(8): 628-638, doi: 10.1038/ nrm2455, indexed in Pubmed: 18628784 